\title{
RESIDUOS DE PARAQUAT EM GRAO DE SOJA QUANDO USADO COM DESSECANTE DA CULTURA
}

\author{
Almeida, F.S. de 1 \\ Pineda-Aguilar, A. 2 \\ Rodrigues, B.N.3 \\ 1 \\ Enge Agre, Fundação Inst. Agronômico \\ do Paraná - IAPAR. Caixa Postal 1331 \\ Londrina-PR. \\ 3acharel em Matemática, M.Sc, IAPAR \\ Eng? Agro, M.Sc., IAPAR.
}

\section{RESUMO}

O uso de paraquat na dessecação da soja deixa resíduos no grão cujo teor está relacionado com a fase de maturação da cultura quando da aplic ação do produto e o intervalo de tempo entre esta operação e a colheita. A lei brasileira estipula como limite de resíduo no grão, $0,1 \mathrm{mg} \mathrm{kg-}^{1} \mathrm{e}$ o prazo de carência de sete dias entre a aplicação e a colheita. Para que esse teor médio de $0,1 \mathrm{mg} \mathrm{kg}^{-1}$, com oscilações de 0,03 a $0,32 \mathrm{mg} \mathrm{kg}-1$, seja atingido, a necessário, não só que a aplicação do paraquat se faça com essa antec edência à colheita como, também, que não ultrapasse a fase de maturação da soja correspondente a $80 \%$ de vagens marrons.

PALAVRAS-CHAVE: Glycine max, maturação, colheita, paraquat.

\section{SUMMARY}

PARAQUAT RESIDUES IN SOYBEAN GRAIN WHEN USED ON THE CROP DISSECATION

The use of paraquat in the soy bean dissecation leaves residue in the grain wich content is related with the crop maturity at the time of application of the product and the time interval between this ope ration and the crop harvest. The brasilian law estipulates a content lower that $0,1 \mathrm{mg} \mathrm{kg}^{-}{ }^{1}$ and an interval of seven days, between ti me of application and harvest. In order to get a medium content of $0,1 \mathrm{mg} \mathrm{kg}^{-}{ }^{1}$, with oscillations of 0,03 to $0,32 \mathrm{mg} \mathrm{kg}^{-1}$, it is necessary to do the paraquat applica tion with that interval of time to harvest but, also, that the crop maturation be lower than the correspondent to $80 \%$ of brown pods.

KEYWORDS: Glycine max, maturation, harvest, para quat.

\section{INTRODUÇÃO}

Os agricultores procedem à dessecaçao da soja (Glycine max (L.) Merrill), como operação de pré-colheita, quando as lavouras se en contram infestadas, com a finalidade de matarem as plantas daninhas e provocarem a desfolha da cultura. A operação facilita o trabalho das colheitadeiras e permite a antecipação da colheita, reduzindo, desta forma, os prejuízos provocados 
por fungos e insetos que incidem sobre a soja no final do ciclo. Utilizam para tal, entre outros, o paraquat, um produto altamente tóxico, com $\mathrm{DL}_{50}$ oral aguda de $150 \mathrm{mg} \mathrm{kg}^{-1}$. Por isto, esta prática cultural tem sido criticada pois, como a operação se realiza próximo à colheita, existe a possibilìda de do produto ser absorvido e concentrar-se no grão, constituindo perigo para o con sumidor.

Esta hipótese tem sido contestada devido à baixa mobilidade do produto dentro da planta. Apesar de ser rapidamente absorvido pelos tecidos vivos a translocação é diminuta, uma vez que, na célula, a molécula é reduzida, pela ação fotossintétíca, a radical livre e, tão $\log o$ formado, novamente oxidado pelo oxigênio molecular, formando-se no processo o peróxido de hidrogênio. Este, concentrando-se na célula, mata-a, bloqueando, des ta forma, a sua via de translocação dentro da planta. Além disso, na época em que se realiza a dessecação da soja, as plantas encontram-se no final do ciclo, com grande parte das folhas e outros órgãos secos, ou próximo de tal ponto. Por isso, a absorção é muito limitada. Também, o vigor vegetativo das plantas é baixo e, portanto, a circulação da seiva no xilema é reduzida. Como o paraquat se transloca por via apoplástica a movimentação é comprometida (8). Finalmente, o produto é fortemente adsorvido pelos colóides e, quan do aplicado sobre tecidos mortos, fica retido na superfície. É nes te estado que e sensível à foto de composição (2) degradando-se por fotólise.

Com base nestes conceitos o fabricante (Imperial Chemical Industries, ICI) defende a teoria de que a contaminação do grão de soja pelo paraquat se dá, não por translocação do produto dentro da planta, mas sim por contato com os tecidos mortos, tanto da soja como das plantas daninhas, durante $o$ processo de colheita e debulha, Por isso recomenda um intervalo de sete dias entre a operação de dessecação e a colheita para dar tempo a que o produto se degrade por fotólise, O Ministério da Agricultura do Brasil estipula este mesmo intervalo de segurança de sete dias e estabelece como limite máximo de resíduo no grão $0,1 \mathrm{mg} \mathrm{kg}^{-}{ }^{1}$. Este é também o limite admitido pela WHO /FAO (6).

Vários pesquisadores $(1,4,5)$, têm-se dedicado ao estudo da residualidade do paraquat na semente de soja, quando usado como dessecante da cultura, relacionando-a, não só com esse intervalo, como também com a fase de maturação em que se encontram as plantas quando da realização da operação. Os resultados sao, porém, pouco consistentes e, por vezes, contra ditórios.

\section{MATERIAL E MÉTODOS}

Procedeu-se à dessecação da soja em 24 lavouras, de sete municípios do Norte e Centro do Paraná (Rolândia-8; Ponta Grossa-4; Faxinal-3; Londrina-3; Cambe3; Arapond as-2; Primeiro de Maio-1). Em todas as lavouras, a densidade de infestação da cultura era alta. Usou-se a dose de 0,4 ha- ${ }^{1}$ de paraquat e, como adjuvante, o Agra $1,0,1 \% \mathrm{v} / \mathrm{v}$, exceto em cinco locais onde foi de 0,30 a $0,32 \mathrm{~kg} \mathrm{ha}^{-1}$.

$\mathrm{Na}$ aplicação utilizou -se sempre o equipamento do agricultor em áreas de alguns hectares, exceto 
em quatro onde se usou pulveriza dor de precisão, tendo-se reduzido a área para 0,1 hectare. $\mathrm{O}$ volume de calda aplicado variou em conformidade com o equi pamento, de $251 \mathrm{ha}^{-1}$, em um caso de aplicação aérea, a 125-350 1 ha- ${ }^{1}$, na mai oria das aplicaçõ es terrestres.

A soja encontrava-se em diferentes fases de maturação, desde $20 \%$ a $100 \%$ de vagens marrons, tendo-se determinado esta percentagem em 10 plantas escolhidas ao acaso, na áre a tratada.

$\mathrm{O}$ intervalo entre a dessecação e a colheita variou entre 2 a 21 dias, respeitando a conveniência do agricultor. $\mathrm{Na}$ colheita utilizou-se a colheitadeira do proprietário, recolhendo-se duas amostras na boca de saída da máquina, quan do se encontrava no terço médio e final da área tratada. Depois de misturadas, foram retiradas cinco amostras das quais, novamente homogeneizadas, tomaram-se duas de $2 \mathrm{~kg}$, sendo uma enviada ao laboratório da ICI, em Jealott's Hill, Inglaterra, para analise de resíduo de paraquat no grão e outra cons ervada no TAPAR. Nos quatro casos de aplicação em área de 0,1 ha, a colheita assim como a debulha foram manuais.

$\mathrm{Na}$ analise de resíduo de paraquat no grão de soja o laboratório usou o "Método Analítico de Resíduos da Plant Protection $\mathrm{n}^{\mathrm{o}}$ 1A" (7) modificado pela inclusão de uma operação de limpeza e remoção de óleo. 0 limite de detecção do método é de $0,02 \mathrm{mg} \mathrm{kg}-1$.

Uma vez obtidos os resultados das análises, agruparam-se os dados em conformidade com o teor de paraquat no grão em: $<0,05 ; 0,06$ g a 0,$10 ; 0,11$ a 0,20 ; 0,21 a 0,$30 ;>0,31 \mathrm{mg} \mathrm{kg-}^{1}$. Dentro de cada grupo sorteou-se uma amostra, retira - da da reserva arma zena da no TAPAR, que se enviou ao laboratório "Morse Labo ratories", em Sacramento, Califórnia, E.U.A., para confirma ção dos dados do lab ora tório da ICI.

$\mathrm{Na}$ análise dos dados usou-se o método de regressão descrito por Drapper \& Smith (3).

\section{RESULTADOS}

No Quadro 1 compararam-se os resultados das análises realizadas no laboratório da ICI com os do Morse Laboratories. Se bem que não coincidentes, as diferenças são pouco acentuadas, a no ser na amostra 5, em que o teor de paraquat determinado pelo laboratório da ICI é bem superior ao do Morse Laboratori es. Por essa razão e, também, porque o dado é discordante em relação aos restantes obtidos por aquele mesmo laboratório, como se pode observar no Quadro 2, resolveu-se descartá-lo. Pela observação desta tabela nota-se que existe tendência para aumento do teor de resíduo à medida que a dessecação se realizou com a soja numa fase de maturação mais adiantada, medida pela percentagem de grãos marrons, e também quando se redu ziu o intervalo entre essa operação e a colh eita.

Estatisticamente o comportamento do teor de resíduo de paraquat no grão $(\mathrm{R})$ em relação ao intervalo da dessecação a colheita (IDC), e descrito pela função de regressão:

$$
\begin{aligned}
& \mathrm{R}=0,6966 \times \mathrm{IDC}^{-1,2314} \\
& \quad(\mathrm{r}=-0,77 * *) \\
& \mathrm{e}, \text { em relação à percentagem de vagens } \\
& \text { marrons } \quad(\mathrm{PVM}) \text { pela } \\
& \mathrm{R}=0,13849 \mathrm{x} \quad 1,024922^{\mathrm{PV}} \mathrm{M} \quad\left(\mathrm{r}=0,67^{*}\right)
\end{aligned}
$$


Quad ro 1. Resíduos de paraquat $\left(\mathrm{mg}_{\mathrm{kg}}{ }^{1}\right)$ de terminado no grão de soja pe $=1$ o lab or at ór io da IC I e da Morse Labora to ri es.

\begin{tabular}{rccc}
\hline $\begin{array}{c}\text { No da } \\
\text { amostra }\end{array}$ & ICI & $\begin{array}{c}\text { Morse } \\
\text { Laboratories }\end{array}$ & Diferença \\
\hline 18 & 0,03 & 0,06 & $+0,03$ \\
23 & 0,08 & 0,11 & $+0,03$ \\
6 & 0,14 & 0,12 & $-0,02$ \\
13 & 0,21 & 0,14 & $-0,07$ \\
5 & 0,43 & 0,24 & $-0,19$
\end{tabular}

Para pos sibilitar os cál culos, atribuiuse o valor de 0,01 a todos os teores de resídu os $<0,02 \mathrm{mg} \mathrm{kg}^{-1}$.

Comple mentan do o estudo proce deuse à determinação da região de previsão para os valores experimentais dos teores de resíduos, à pro habilidade de $80 \%$. Fizeram-se os aju stes utilizando a média dos teo res, quando havia mais do que um, para o mes mo intervalo â colheita ou fase de maturação.

Nas Figuras 1 e 2 têm-se a representação gráfica dessas equações.

Deduz-se deles que, proceden do à dessecação com a soja na fase de maturação máxima de $80 \%$ de vagens marrons, pode esperar-se que o resíduo na semente seja de $0,1 \mathrm{mg} \mathrm{kg}^{-1}$, com oscilações que, à probabilidade de $80 \%$, poderão ir de 0,03 a $0,32 \mathrm{mg} \mathrm{kg}^{-1}$. Estes resultados confirmam os obtidos por BASTIDAS et al. (1), Durigan (4) e Durigan \& Cattaneo (5), já mencionados. Respeit an do um in tervalo mínimo de 5 dias entre a data de dessecação e a colheita, o te or de resíduo também de $0,1 \mathrm{mg} \mathrm{kg}^{-1}$, com os limites de previsão de 0,03 a $0,28 \mathrm{mg}$ $\mathrm{kg}^{-1}$, a mesma probabilidade de
$80 \%$, o que se integra no intervalo de segurança de sete dias estipulado pelo Ministério da Agricultura.

O processo pode ser explicado pela característica do produto só ser absorvido e translocado pelos tecidos vivos e em plantas em plena atividade vegetativa, ficando, nos órgãos mortos, adsorvido a eles, estado em que e sensível à fotodecompo sição. Procedendo ides secação com a soja em estado avançado de maturação não é absorvido, ficando retido na sua superfície e, quando da colheita, contamina, por contato, a semente. Encontrando-se a soja menos madura, com tecidos ainda vivos, é absorvido mas sendo a translocação reduzida, não atinge o grão. $\mathrm{O}$ produto adsorvido fica exposto à luz solar e é degradado por fotólise, o que explica que após o intervalo de cinco dias entre a dessecação e a colheita, grande parte se encontre degradado por fotólise e a contaminação do grão seja menor, em média, do que $0,1 \mathrm{mg} \mathrm{kg}-^{1}$ exigido por lei.

$\mathrm{Na}$ prática os dois processos acumulam-se uma vez quanto mais ma dura se encontra a soja quando da dessecação, mais rapidamente o agri- 
Quadro 2. Teores de paraquat $\left(\mathrm{mg} \mathrm{kg}^{-1}\right)$ no grão, agrupados pela fase de maturação da soja quando da dessecação (\% de vagens marrons) e intervalo até a colheita, determinados pelo laboratório da ICI

\begin{tabular}{|c|c|c|c|}
\hline \multicolumn{2}{|c|}{ Fase de maturação } & \multicolumn{2}{|c|}{ Intervalo à colheita } \\
\hline $\begin{array}{c}\text { Vagens marrons } \\
\%\end{array}$ & $\begin{array}{l}\text { Residuo } \\
\mathrm{mg} \mathrm{kg}^{-1}\end{array}$ & Nọ de dias & $\begin{array}{l}\text { Resíduo } \\
\text { mg kg-1 }\end{array}$ \\
\hline $\begin{array}{r}100 \\
100 \\
100 \\
100 \\
100 \\
100 \\
92 \\
90 \\
80 \\
80 \\
75 \\
70 \\
70 \\
70 \\
70 \\
70 \\
70 \\
70 \\
60 \\
60 \\
60 \\
30 \\
20\end{array}$ & $\begin{array}{r}0,43 * \\
0,28 \\
0,16 \\
0,16 \\
0,14 \\
0,08 \\
0,18 \\
0,28 \\
0,21 \\
<0,02 \\
0,08 \\
0,06 \\
0,03 \\
0,03 \\
0,02 \\
<0,02 \\
<0,02 \\
<0,02 \\
0,03 \\
0,02 \\
<0,02 \\
0,02 \\
0,07\end{array}$ & $\begin{array}{r}2 \\
3 \\
3 \\
3 \\
3 \\
4 \\
5 \\
5 \\
5 \\
5 \\
5 \\
6 \\
6 \\
8 \\
8 \\
9 \\
9 \\
11 \\
11 \\
11 \\
16 \\
17 \\
20 \\
21\end{array}$ & $\begin{array}{c}<0,02 \\
0,43 \\
0,18 \\
0,16 \\
0,08 \\
0,21 \\
0,28 \\
0,28 \\
0,16 \\
0,14 \\
<0,02 \\
0,08 \\
0,03 \\
0,06 \\
0,03 \\
0,03 \\
<0,02 \\
0,02 \\
0,02 \\
0,02 \\
0,06 \\
0,07 \\
<0,02 \\
<0,02\end{array}$ \\
\hline
\end{tabular}

* Dado descartado. Para efeitos de análise dos dados atribuiu-se o valor de 0,01 aos teores de resíduo $<0,02 \mathrm{mg} \mathrm{kg}-1$. 


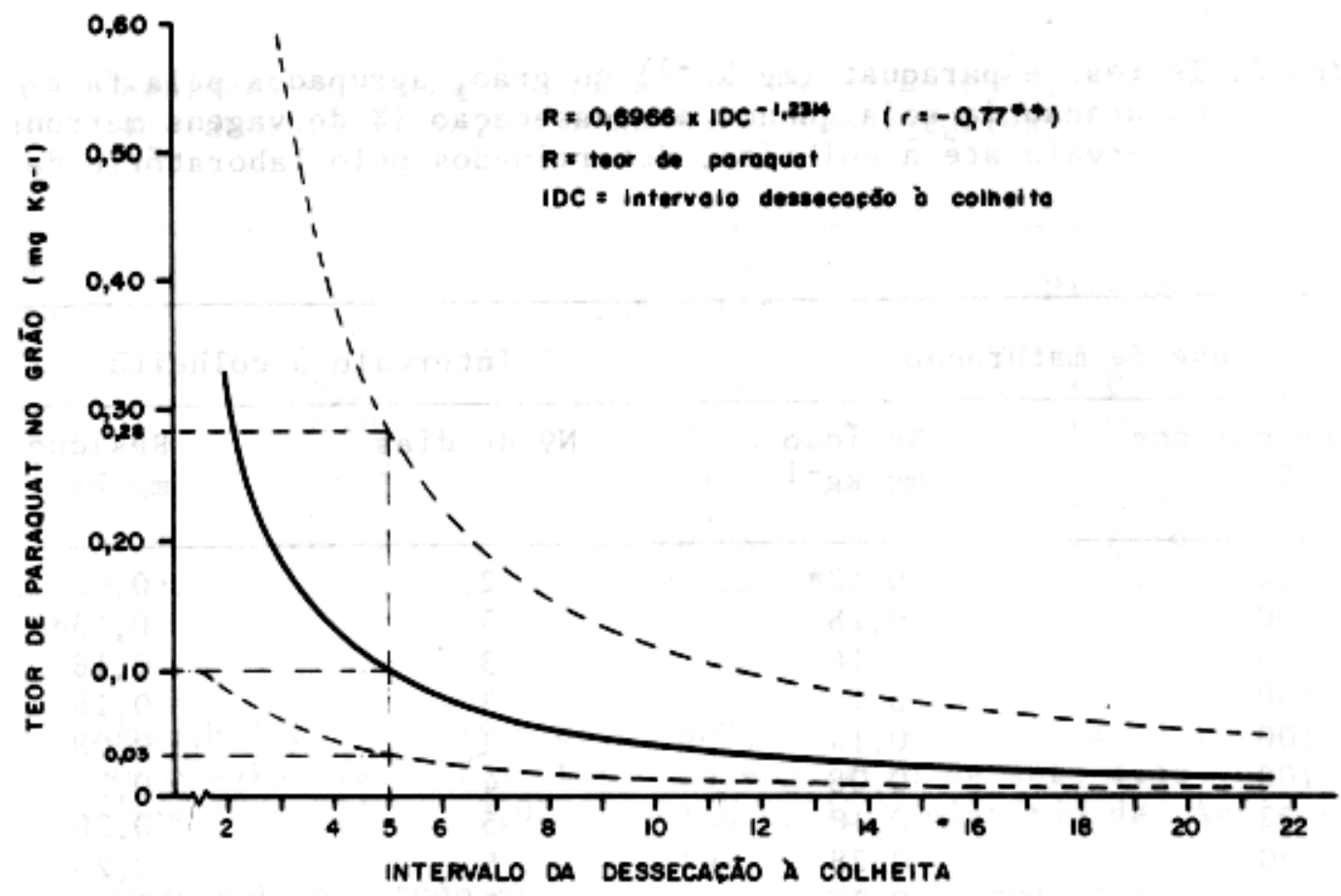

FIG 1 - REGRESSÃO E LIMITES DE PREVISAOO DA VARAAGÃO DO TEOR DE RESÍDUO DE PARAOUAT NO GRĀO DE SOJA (R) EM FUNGĀO DO INTERVALO ENTRE A DATA DA DESSECACÃo E A DA COLHEITA (IDC)

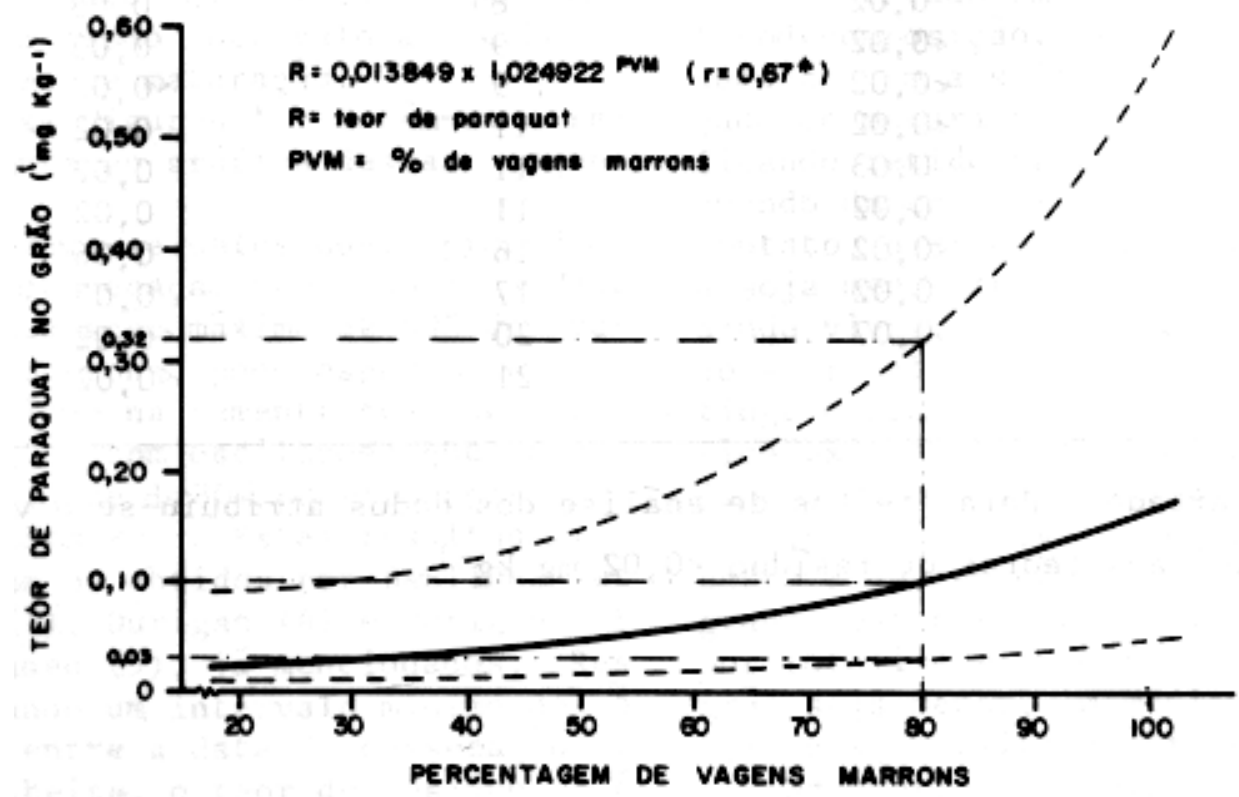

FiG. 2 - REgRESSAL E LIMITES de PREVISÃo dA VARIACAO DO TEOR IE RESÍDUO DE PARAOUAT NO GRĀo DE SOJA (R) EM FUNCĀO DA pERCENTAGEM de VAGENS MARRONS ( PVM) MAS PLANTAS, QUANDO DA DESSECACTO 
cultor procede a colheita, a fim de evitar perda de qualidade e quantidade do grão.

Esta teoria esta devidamente comprovada e requer que seja mais profundamente estudada.

No processo de dessecação da soja com paraquat o teor de produto que fica retido na semente correlaciona-se com a fase de maturação em que se encontra a soja quanto da realização da dessecação e, também, com o intervalo de tempo entre a operação e a colheita.

Procedendo a dessecação com um intervalo mínimo de cinco dias a colheita pode prever-se teores de resíduos de paraquat no grão que, à probabilidade de $80 \%$, oscilem entre 0,03 e $0,28 \mathrm{mg} \mathrm{kg-}{ }^{1}$, com média muito próxima a $0,1 \mathrm{mg} \mathrm{kg-1}$.

Fazendo a dessecação com a soja na fase de maturação corresponde a $80 \%$ de vagens marrons, espera-se que o teor de paraquat, a mesma probabilidade, oscile entre 0,03 e 0,32 $\mathrm{mg} \mathrm{kg}^{-1}$, com média mui to próxima de $0,1 \mathrm{mg}$ $\mathrm{kg}-1$.

O uso do produto deve restringir-se não só ao intervalo de segurança, já estipulado por lei, de 7 dias entre a aplicação e a colhei ta, como também, ao estádio máximo de maturação da soja correspondente a $80 \%$ de vagens marrons.

\section{LITERATURA CITADA}

1. Bastidas, G.; Franco, H.; Cruz, $R$. de la, Defoliantes en soya (Glycine max (L.) Merril). Acta Agronómica, 21 (2): $51-8,1971$.

2. Calderbank, A. The bypyridylium herbicides. Advances in pest control research, 8: $127-235,1968$.

3. Drapper, N.R. \& Smith, H. Applied regression analysis. New York, John Willes \& Sons, 1981. 709p.

4. Durigan, J.C. Efeitos da apli cação em pré-colheita de des secantes em duas cultiva $=$ res de soja (Glycine max (L.) Merril). Haboticabal, SP, Faculdade de Ciências A grärias e Veterinārias, $197 \overline{9}$. (Tege MSc.).

5. Durigan, J.C. \& Cattaneo, S.F. Efeitos da aplicação, em pré-colheita de dessecante em soja (Glycine max (L.) Merril) da cultivar UFV-1. Jaboticabal, SP. Faculdade de Ciências Agrärias e Veterinārias, 1982 .

6. FA0, Roma, Itälia. Pesticides residues in food: Report of 1972 joint meeting of the FAO working party of experts on pesticides residues and of the WHO expert committee on pesticide residue. Roma, 1973 (FAO, Agricultural Stu dies, 90).

7. Imperial Chemical Industries. plant protection residue analitycal method no 1A. Bra cknel1, Inglaterra, Jealot $\bar{t}$ 's Hill Res. Station s.d.

8. Summer, L.A. The bipyridylium herbicides. New York, Academic Press, 1980. 